\title{
A Web Cache Replacement Strategy for Safety-Critical Systems
}

\author{
Jianhai DU, Shiwei GAO, Jianghua LV, Qianqian LI, Shilong MA
}

\begin{abstract}
A Safety-Critical System (SCS), such as a spacecraft, is usually a complex system. It produces a large amount of test data during a comprehensive testing process. The large amount of data is often managed by a comprehensive test data query system. The primary factor affecting the management experience of a comprehensive test data query system is the performance of querying the test data. It is a big challenge to manage and maintain the huge and complex testing data.To address this challenge, a web cache replacement algorithm which can effectively improve the query performance and reduce the network latency is needed. However, a general-purpose web cache replacement algorithm usually cannot be directly applied to this type of system due to the low hit rate and low byte hit rate. In order to improve the hit rate and byte hit rate, a data stream mining technology is introduced, and a new web cache algorithm GDSF-DST (Greedy Dual-Size Frequency with Data Stream Technology) for the Safety-Critical System (SCS) is proposed based on the original GDSF algorithm. The experimental results show that compared with state of the art traditional algorithms, GDSF-DST achieves competitive performance and improves the hit rate and byte hit rate by about $20 \%$.
\end{abstract}

Keywords: data mining; data query; Safety-Critical System; spacecraft; Web Cache Replacement Strategy

\section{INTRODUCTION}

Information technology significantly improves the efficiency of automated testing of the equipment, which leads to an explosive growth of the test data $[1,2]$. In particular, more complex and extensive test data are generated in the comprehensive testing of the complex SCS (safety-critical systems), such as the spacecraft [3]. For each model of SCS, the total amount of comprehensive test data per year can reach the $\mathrm{T}$ order of magnitude, and the test data are various and multidimensional. These data need to be stored in multiple data tables. In each data sheet of a table there are tens of millions of data items [4]. Thus, managing and maintaining the huge and complex test data poses challenges. The primary factor that affects the system performance and the management experience is efficiently querying test data, which is one of the most frequent applications.

In a traditional $\mathrm{B} / \mathrm{S}($ Brower/Serve) data querying system, data is retrieved from the database server for every query. Meanwhile, each query has to retrieve data from the entire database; therefore, a large number of queries from the large database may cause a significant time delay. This approach frequently transmits a large number of the same data between the database server and the application server, resulting in unnecessary network burden. In addition, for the equipment being tested, the database server needs to dynamically load a large amount of test data. The database server is accessed frequently, and thus the performance of the whole system is seriously affected. To ensure the stability of the server and the integrity of the data entry, data query should consume as few resources of the server as possible.

Web caching presents an effective way to solve the aforementioned problems. It plays an important role in improving Web performance by placing probably reaccessible objects closer to users, which can thus reduce the response time, the consumption of network bandwidth, and the workload of the original server [3]. An efficient web cache replacement algorithm for a particular application environment can also significantly affect the system performance and the user experience.
The main criterion for the cache replacement algorithm is to minimize the metrics of average delay, error rate, and byte error rate. In a cache replacement algorithm, the main factors that need to be considered in the value function design include Recency, Frequency, Cost, Size, Expiration Time and Modification Time [6, 7]. Particularly, Recency refers to the last time when the object is referred. Frequency is the total number of times of object reference. Cost refers to the resources' cost of acquiring the object from the original server. Size refers to the size of the object. Expiration Time refers to the expiration time of the object, and Modification Time refers to the time when the object is recently modified.

Considering the above factors, the cache replacement algorithm can be divided into the following five categories [6]. The first type is based on LRU (Least Recently Used), including LRU, LRU-Threshold (Least Recently Used-Threshold), Pitkow/Recker strategy, EXP1 (Exponential 1), Value-Aging, HLRU (History LRU), PSS (Pyramidal Selection Schema) [7], and Partitioned LRU. These algorithms are relatively easy to implement with low time complexity. However, the LRU variants are simple, and unable to effectively consider the effect of the object size or the access frequency. The second type of algorithms is based on LFU (Least Frequently Used) algorithms, including DSLFU (Delay sensitive LFU), LFU-Aging (Least Frequently Used-Aging), LFUDA(LFU with Dynamic Aging), $\sigma$-Aging, swLFU (Server-Weighted LFU), DSLV (Dynamic Semantic LFU Policy with Victim tracer) [8], and ALFUR (Average Least Frequency Used Removal). These algorithms require complex cache management, and can cause cache pollution. There may exist multiple objects with the same frequency count at the same time, in which case additional factors are required to determine which object to remove. The third type of algorithms takes both recency and frequency into account. Some algorithms add other factors as well. These algorithms include LRFU (Least Recently/Frequently Used), SLRU (Segmented LRU), SF-LRU (Second ChanceFrequency-Least Recently Used), Generational Replacement, LRU* (Least Recently Used*), LRU-Hot (Least Recently Used-Hot), LRU-SP (LRU-size-adjusted and popularity-aware), and 
CSS (Cubic Selection Scheme). If designed properly, they can avoid the aforementioned shortcomings of the first two types of algorithms based on recency and frequency respectively. However, because of the special process, most of these algorithms can introduce additional complexity. Only the LRU* algorithms and Generational Replacement algorithms attempt to combine LRU with frequency. Nevertheless, they do not take into account the effect of the object size. The fourth type of cache replacement algorithms is function-based. These algorithms can optimize the performance by selecting appropriate weighting parameters, including GDS (Greedy-Dual-Sizel), GDSF (Greedy-Dual-SizeFrequency), Server-assisted cache replacement, TSP (Taylor Series Prediction), Bolot/Hoschka's strategy, MMetric, Hybrid, LRV (Lowest Relative Value), LUV (Least-Unified Value), and LR (Logistic Regression)Model. They consider multiple factors to deal with different workloads. However, the limitation of these algorithms is that it is difficult for them to choose an appropriate weighting parameter. New problems may arise in the computation of the function values. The fifth type of algorithms uses a random decision to find the object to be replaced [6], including RAND (random), Harmonic, LRU-C (LRU-Cost), LRU-S (LRU-Size), and Randomized replacement with general value functions. These algorithms are easy to implement and do not require any special data structures when inserting objects or deleting objects. However, it is difficult for these algorithms to evaluate them. For example, there may exist slight differences in the results of different simulation instances running on the same Web request trace.

Apart from the traditional cache replacement algorithms summarized above, several more intelligent and adaptive cache replacement algorithms have been proposed in recent years. The basic principles of these intelligent algorithms are as follows. A machine learning model (mostly classifier, such as a SVM (Support Vector Machine) [9, 10], a NB (Naive Bayes classifier), or a C4.5 (decision tree) and so on) is first trained through the contents of the Web log for classifying the incoming web objects. Then based on these classified results, traditional web caching replacement algorithms deal with new web objects and decide which object will be replaced when the cache is not enough. Therefore, the performance of the traditional web caching alternative algorithms is still very important. In particular, a SmartCache is designed for a router-based system [11], which is composed of the cache, the SVM trainer and classifier, and the browser extension. The browser interacts with users to collect their experience satisfaction and prepare training dataset for the SVM trainer. With the desired features extracted from training dataset, the trained SVM classifier is used to predict classes of the Web objects. Then, in conjunction with the LFU, the cache makes a cache replacement based on the SVM-LFU policy. In recent studies [12, 13], Ali Waleed, et al., proposed a series of intelligent caching replacement approaches known as SVM-LRU, SVMGDSF, NB-LRU, NB-GDS, NB-DA, C4.5-GDS and C4.5-LRU by integrating machine learning models, such as a SVM, a NB, or a C4.5, with conventional Web proxy caching techniques, such as LRU, GDS and GDSF. A learning based replacement algorithm (LBR) is proposed to build an efficient replacement model for web caching by incorporating a machine learning technique (naive Bayes) into the LRU replacement method to improve the prediction of possibility [14]. Fuzzy Similarity is integrated with traditional caching algorithms for caching replacement strategies [15]. A semi-intelligent approach is developed for web cache replacement using a multinomial web object classifier [16]. A Neural Network Proxy Cache Replacement (NNPCR) method is proposed to use neural networks to decide which objects are worth caching or being replaced by other objects [17]. Three replacement policies are introduced by following different strategies, including a dynamic programming approach, a branch and bound approach, and a heuristic approach, to find the best objects to be kept and the worst objects to be replaced, as well as to efficiently select objects to be evicted, respectively [18]. Specific cache replacement strategies have been improved and designed for specific fields in recent studies. Particularly, an enhanced method is proposed to actively cache the data for data-intensive computations in distributed GIS by considering both data relationships and their timeliness [19]. A novel cache scheme, named Optimized Cache Replacement, is proposed for Information Centric Networks (OCRICN) [20]. For Network-Coding-Assisted Data Broadcast, a decoding-oriented cache management scheme, named decoding-oriented least recently used (DLRU), is proposed to incorporate decoding information in making cache replacement decisions [21].

However, these existing intelligent algorithms above have some common shortcomings. For instance, to achieve desirable performance for the intelligent algorithms, they usually need more system resources, a large number of data for training in advance and higher computational complexity. Thus, the systems based on these algorithms have low cost performance and mainly aim at ordinary users access web pages, multimedia, etc. in the World Wide Web. In addition, each type of algorithms has its own advantages and disadvantages. Even if some are the ones so-called "good enough" through which better results can be obtained on the basic performance indicators $[6,22]$, none can perform well in any application environment. The network environment is dynamic and uncertain. For instance, workload varies with different applications; a variety of Web access features exist and design goals for different cache systems and the design factors to be considered are not the same. No replacement strategies can well adapt to any network situation. Therefore, it has become a hot spot in the field of cache replacement which algorithm is the most appropriate replacement strategy according to varied network conditions and access characteristics [22].

Spacecraft integration test data are time-related, timeintensive, of large volume and complex structures. The users' query behaviors on the data are of strong temporal locality, strong spatial locality, high frequency (a small part of objects are accessed many more times than the others) and multiple visits (in order to ensure the completeness and the accuracy of the test, each data is accessed multiple times) [23]. Nevertheless, the existing web cache replacement algorithms are not suitable for integrated test data query systems for safety critical systems like Spacecraft. For this kind of system, a new 
web cache replacement algorithm needs to be designed to improve the cache hit rate of the algorithm, and consequently realize the higher utilization rate of the resources on the database server, less network load, a better overall performance of the system, better user experience and the higher work efficiency of users.

This study proposes a Web cache replacement algorithm based on GDSF algorithm, GDSF-DST (Greedy Dual-Size Frequency with Data Stream technology). The algorithm is proposed by analyzing the characteristics of test data of the safety-critical system and users' query behaviors. Considering the characteristics of practical application requirements and the current classical Web cache replacement algorithms, this algorithm also introduces technology related to data stream mining and adopts the idea of sliding time window. With comprehensive experiments and analysis, the results show that the algorithm presents better performance, with less consumption of system resources, higher efficiency, stability and certain universality.

\section{BACKGROUND}

\subsection{The characteristics of Spacecraft Integrated Test Data and the Query Behaviours of Users}

In the spacecraft integrated test database system, a variety of test data are stored in various types of database tables. The typical spacecraft integrated test data table structure includes the following characteristics. Taking time as the primary key, the comprehensive test data store the value [24] of each parameter at this time point. Specifically, the integrated test data of spacecraft have the following four characteristics.

(1) Large volume of data: About $20 \mathrm{G}$ of data are produced per hour, and the amount of data produced per day can be up to $480 \mathrm{G}$. For a single spacecraft model, the total amount of its data can reach $\mathrm{T}$ orders of magnitude.

(2) Complicated data structures: The data types of spacecraft integrated test data are numerous. The subsystems each spacecraft model belongs to are various, and each subsystem has different parameters. So the data structures are very complicated. These complex data are stored in multiple database tables.

(3) The data are associated with time: In the database, the business data take time as the key. Logically, the data are time-correlated, that is, the time information is used as the retrieve condition when data are queried and analyzed. It emphasizes on the situation of the data at each time point in a certain period and the changing trend of the data throughout the whole time period.

(4) Time intensive: One piece of datum or more are produced per second. Even if the data within a relatively short period of time are queried, there is still a large number of query results. For example, query data within an hour are at least 3600 pieces.

From a macro point of view, users have some behavioral characteristics in querying the data of spacecraft integrated test system, which mainly include the following four aspects.

(1) The data queried by users are of strong temporal locality: For the case of the data query with a device being tested, the data queried in a particular day are usually limited to the data generated on that day, and thus the queried data extent evolves as the day goes by. For the case of the summary query, the data queried daily are generally limited to a period of time (1 week to 10 days) in the previous test phase, and the data are usually summarized and analyzed according to a certain time period.

(2) Users repeatedly visit the data: In order to ensure the integrity and the accuracy of the test, each datum will be queried many times.

(3) The data queried by users are of spatial locality: All the integrated test data of the current models are stored in the database server. However, the data queried by users are limited to a short test time. Therefore, being a small part of the data in the database, the accessed data are of spatial locality.

(4) Some objects are accessed frequently: the frequency of some objects accessed over a certain period of time is significantly larger than that of the others. For example, the part with a fault will be repeatedly checked by some staff.

\subsection{Problem analysis}

A query system is a Web application of $\mathrm{B} / \mathrm{S}$ architecture in which data access is incentive; that is, each front-end operation will have a corresponding response. Due to the confidentiality of the spacecraft system, data backups are forbidden on an ordinary PC. And the large result set makes it unfeasible to cache the results on the browsers. Therefore, for each query operation, the results must be returned from the background. Based on the previous analysis of the spacecraft integration test data and the query characteristics of users, with the method of the Web server caching data, this algorithm successfully solves the problem that the same data have to be repeatedly transmitted between the browsers and the database, reducing the access load of the database server and the network traffic user query response time. Those methods referred to above are not perfectly suitable for spacecraft integrated test data query in a real-time environment. The method proposed in this paper is that all the data accessed by users within a business day are considered as an accessing scale unit. The time interval for accessing data within a business day is represented as timeInterval $=[$ begin, end $]$. In order to ensure the continuity of the data in the cache, the data in the timeInterval in each original database table are logically divided into different small data tables by the hour. Then data can be obtained from the database server on a small data table scale. And each small data table is viewed as an object to be cached on the Web application server. Consequently, the core problem is that excellent cache replacement algorithms need to be designed to improve the performance of the system.

Depending on different locations in the Web, the Web cache can be categorized as a browser cache, a Web proxy cache, and an original server cache. The browser cache is on the client side, the original server cache is the cache of the server itself, and the Web proxy cache is on the Web proxy server. The Web proxy server lies between the user receiver and the original server, receiving requests from hundreds of users and replying the results to users. The Web proxy cache is widely used in Web 
proxy servers, the performance of which has a direct influence on that of Web access. It is exactly the focus in this paper. Spacecraft system is a typical safety critical complex system. The common methods given in previous literature are not completely applicable to the spacecraft integrated test data query in a real-time environment. Therefore, it is of great significance to design an excellent cache replacement algorithm to improve the performance of the system.

\section{GDSF ALGORITHM}

GreedyDual algorithm was first proposed in the paper [25], and then based on GreedyDual algorithm, Pei Cao and Sandy Irani proposed GreedyDual-Size (GDS) algorithm [26]. The literature [27] proposed GDSF algorithm on the basis of GDS algorithm, which introduced the influence of the times of object visit on the performance of cache replacement algorithms. At present, GDSF algorithm, one of the most widely used cache replacement algorithms, is universally recognized as a "good enough" cache replacement algorithm with a better cache hit rate. It takes into account the access frequency of Web objects, the cost of acquiring objects and the influence of object sizes. By setting up the aging factor, the influence of the Web access temporal locality on cache replacement is perfectly fused into the algorithm. Whereas unable to adapt to the rapid changes of data stream, GDSF algorithm fails to get enough good cache hit rate. Web application is a typical data stream application [28], and Spacecraft integrated test data query system is a type of Web application. On the basis of GDSF algorithm, GDSF-DST algorithm, GDSF with Data Stream Mining, is put forward, with some methods used to mine frequent patterns on time sliding window in data stream mining. Improving the frequency counting method of GDSF and bringing in the concept of expired transaction, the algorithm is of better adaptability, cache hit ratio and performance of cache system.

The cache replacement algorithm GDSF uses the following function to calculate the eigenvalue of each Web object:

$$
V(\boldsymbol{I})=L+f_{\text {freq }}(\boldsymbol{I}) * \frac{\operatorname{Cost}(\boldsymbol{I})}{\operatorname{Size}(\boldsymbol{I})}
$$

where:

- $\quad I$ is a Web object.

- $\quad V(\boldsymbol{I})$ is the eigenvalue of the Web object $\boldsymbol{I}$.

- $\quad L$ is an aging factor whose initial value is 0 . When cache replacement occurs, the value of $L$ equals the eigenvalue of the latest replaced object.

- $f_{\text {freq }}(\boldsymbol{I})$ represents the frequency count of the object $\boldsymbol{I}$, that is, the times the object has been requested before.

- When object $\boldsymbol{I}$ is hit by cache, its corresponding value is incremented by 1 , otherwise $f_{\text {freq }}(\boldsymbol{I})$ is equal to 1 .

- Size $(\boldsymbol{I})$ refers to the size of the object $\boldsymbol{I}$.

- $\operatorname{Cost}(\boldsymbol{I})$ stands for the cost of retrieving the object $\boldsymbol{I}$.

The pseudocode of the algorithm GDSF is shown in Tab. 1. When a cache replacement is needed, GDSF replaces the object with the smallest eigenvalue. It is obvious in Eq. (1) that the GDSF algorithm takes into consideration the influence of the object access frequency $\left(f_{\text {freq }}(\boldsymbol{I})\right)$, the cost of retrieving the object $(\operatorname{Cost}(\boldsymbol{I}))$ and the object size $(\operatorname{Size}(\boldsymbol{I}))$. In addition, GDSF also takes into account the temporal locality of object access. So each time the object in the cache is hit, the eigenvalue $V$ of the object will be updated. And when the cache replacement occurs, the value of the aging factor $L$ is updated to the maximum $\mathrm{V}$ value in the eigenvalues of those evicted objects. Consequently, the value of the aging factor $L$ is increasing gradually. By this way, the aging factor part in the eigenvalue function of the recently hit object is larger than that in the eigenvalue function of the object not having been accessed for a long time. And the influence of the temporal locality feature of the object access is integrated into the algorithm accordingly. Yet, it is beyond GDSF that users are more concerned about recent transactions and those older transactions have little effect on the transactions currently queried. However, it is rather costly to deal with those older ones. So the transaction data in a certain period of time may be of more significance. The application of sliding window model can just cope with the problem of data expiration. Hence, the algorithm GDSF-DST will be presented in the next section.

Table 1 GDSF algorithm

cache replacement algorithm GDSF: $\operatorname{GDSF}\left(I_{1}, I_{2}, \ldots, I_{z}\right)$

Input: The sequence of objects requested: $I_{1}, I_{2}, \ldots, I_{z}$

Output: The processing of each object by the cache replacement algorithm, and then return the requested object sequence.

1. Initialize $L \leftarrow 0$;

2. For each $I_{n}$ in $I_{1}, I_{2}, \ldots, I_{z}$

3. If $I_{n}$ is already in cache then

4. $V\left(I_{n}\right) \leftarrow L+f_{\text {freq }}\left(I_{n}\right) * \frac{\operatorname{Cost}\left(I_{n}\right)}{\operatorname{Size}\left(I_{n}\right)}$;

5. End If;

6. If $I_{n}$ is not in memory then

7. While there is not enough room in cache for $I n$;

8. Evict $I_{e}^{j} ; / / I_{e}^{j}$ is the minimum $V$ value of all the object in cache

9. End while;

10. $L \leftarrow V\left(I_{e}^{k}\right) ; / / I_{e}^{k}$ is the maximum $V$ value of all the object evicted recently

11. End If;

12. bring $I_{n}$ into cache ;

13. $V\left(I_{n}\right) \leftarrow L+f_{\text {freq }}\left(I_{n}\right) * \frac{\operatorname{Cost}\left(I_{n}\right)}{\operatorname{Size}\left(I_{n}\right)} ;$

14. End For:

15. return the requested object sequence;

\section{GDSF-DST \\ 4.1 Related definitions}

Before the explanation of the algorithm, the concepts to be used in it are introduced firstly.

Let $\boldsymbol{I}=\left\{I_{1}, I_{2}, \ldots, I_{n}, \ldots, I_{m}\right\}$ be a set of data items.

Definition 1: transaction $T_{j}=\left\{I_{x}, I_{y}, \ldots, I_{z}\right\}$ is a subset of $\boldsymbol{I}$, namely, $T_{j} \subseteq \boldsymbol{I}$. And each transaction has its own identifier called TID. The data items in the transaction are out of order and can be arranged in any order.

Definition 2: data stream $D S=\left\{T_{1}, T_{2}, \ldots, I_{j}, \ldots, T_{m}\right\}$ consists of transactions sequentially arranged in time order. The number of transactions may be infinite. The 
TID of each transaction is assigned in terms of the arrival time and it is unique.

Definition 3: The sliding window SW [29] is the sequence of the most recent $N(N \geq 0)$ transactions in the data stream $D S$. The order of the transactions in the sliding window is consistent with that in the data stream $D S$. Depending on whether $\mathrm{N}$ is fixed or variable, the sliding window can be classified into fixed-sized sliding window and variable-sized sliding window. The commonly used variable-sized sliding window is mainly based on the time sliding window, which means that transactions for the most recent time period (e.g. 1 hour) are saved in the window. The operation in sliding windows involves deleting the oldest transactions from a sliding window and adding new ones to it. When the number of transactions in the window reaches $N$, removal and insertion in a fixed-sized sliding window must be simultaneous, while it is unrestrained in a variable-size one [30].

In this paper, a transaction represents a set of the objects requested by a user with a Web click. Each data item table in the set denotes a Web object. The data stream composed of all the transactions in order of arrival is the Web click stream which will be analyzed in the following section.

\subsection{GDSF-DST Algorithm Description}

The eigenvalue function of the algorithm GDSF-DST can be expressed as:

$$
\left\{\begin{array}{l}
V\left(I_{n}, T_{j}\right)=L+f_{\mathrm{d}}\left(I_{n}, T_{j}\right) * \frac{\operatorname{Cost}\left(I_{n}\right)}{\operatorname{Size}\left(I_{n}\right)},\left(T_{\text {oldest }} \geq T_{j} \leq T_{\text {last }}\right) \\
V\left(I_{n}, T_{j}\right)=0,\left(T_{\text {oldest }}<T_{j}, T_{j} \leq T_{\text {last }}\right)
\end{array}\right.
$$

Where:

$$
\begin{gathered}
f_{\mathrm{d}}\left(I_{n}, T_{j}\right)=\left\{\begin{array}{cc}
r & \text { if } j=1 \\
f_{\mathrm{d}}\left(I_{n}, T_{j-1}\right) * f+r & \text { if } j \geq 2
\end{array}\right. \\
r=\left\{\begin{array}{cc}
1 & \text { if } I_{n} \in T_{j} \\
0 & \text { if } I_{n} \notin T_{j}
\end{array}\right.
\end{gathered}
$$

In Eq. (2), the meanings of $L, \operatorname{Cost}\left(I_{n}\right)$ and $\operatorname{Size}\left(I_{n}\right)$ are the same as those in formula (1). $V\left(I_{n}, T_{j}\right)$ represents the eigenvalue of object $I_{n}$ when transaction $T_{j}$ arrives. $f_{\mathrm{d}}\left(I_{n}, T_{j}\right)$ denotes the decay support amount of object $I_{n}$ when transaction $T_{j}$ arrives. $T_{\text {oldest }} \geq T_{j} \leq T_{\text {last }}$ and $T_{\text {oldest }}<T_{j}$ or $T_{j} \leq T_{\text {last }}$ represent the sliding time window, which introduces the impact of expired transactions on the algorithm.

Web object access is of temporal locality, namely, the closer from now the object is accessed, the more likely it is to be accessed in the future. In other words, the Web click stream is bound to change over time, and commonly the information contained in recently generated transactions is more valuable than it in historical transactions [31]. Therefore, the time decay model support amount algorithm [32, 33] is proposed to gradually lower the impact of historical transactions. The decay ratio of data items in unit time is decay factor $f(0<$ $f \leq 1)$. When transaction $T_{j}$ arrives, the decay support amount of data item $I_{n}$ is $f_{\mathrm{d}}\left(I_{n}, T_{j}\right)$. Finally, the decay support amount of data item $I_{n}$ can be calculated by Eq. (3).

\subsection{Algorithm Flow of GDSF-DST}

Before introducing the algorithm flow, the data structure to be used in the algorithm is given first:

A sliding time window is a time-based one able to store the ordered transactions arriving in the most recent variable period (time). The members of the sliding time window are represented by a data structure called SwItem. Fields include a submission time subTime and a transaction TID. When a new transaction $T_{j}$ arrives, the sliding time window requires updating. Encapsulate its fields into SwItem, insert the SwItem into the back of the queue, check from the beginning of the queue and then delete all the expired transactions. oldestTid and newestTid respectively represent the transaction TID in the head of the sliding time window queue and the one in the end of the queue (newestTid minus oldestTid equals the length of the sliding window (time)).

fdHash refers to object information Hash table, which is used to store the decay support amount of Web objects and other related information and expressed in the form of key value pairs. Given the Web object name is the primary key, the value is a data structure called Item_Node, <Item_Name, Item_Node $>$. Item_Node contains five data fields: tid (the TID of the latest transaction consisting of object Item_Name), count (the decay support amount), size (the object size), value (the object eigenvalue) and cost (the cost of gaining the object from the original server). Recording tid is to judge whether a transaction is an outdated one, and to calculate the decay support amount of the object as well.

When a new transaction $T_{j}$ arrives, the GDFS-DSM algorithm flow for each object $I_{n}$ in the transaction is depicted as follows:

If object $I_{n}$ exists in the cache and is hit, the decay support amount of the object $I_{n}$ is updated:

count $=$ count $* f^{j-t i d}+1$

(The decay support amount count has the same meaning as $f_{\mathrm{d}}\left(I_{n}, T_{j}\right)$ in Eq. (2). $f$ is the decay factor, which equates with that in Eq. (3). The range of $f$ is $(0,1]$. When the value of $f$ is 1 , no decay happens.)

Update the TID recently contained by the object $I_{n}$ : tid $=j$; update the eigenvalue $V\left(I_{n}, T_{j}\right)$ of the object $I_{n}$ by Eq. (2); and the value of Used remains unchanged. Then the processing of the object ends in the algorithm.

If the object $I_{n}$ does not exist in the cache, the decay support amount of the object $I_{n}$ equals 1 : count $=1$; The TID of the transaction recently containing the object $I_{n}$ is $j$ : tid $=j$.

Compute the eigenvalue $V\left(I_{n}, T_{j}\right)$ of the object $I_{n}$ by Eq. (2) and save the relevant information of the object $I_{n}$ to the object information hash table fdHash:

Used $=$ Used $+\operatorname{Size}\left(I_{n}\right)$ 
If Used $\leq$ TotalSize, there is adequate space in the cache to save the object $I_{n}$. In this case, store the object $I_{n}$ into the cache directly. The process of the object is over.

If Used > TotalSize, the cache space is inadequate. In this case, a cache replacement is required. So expired objects need picking out one by one in fdHash. (As to an Item Node in fdHash, if Item_Node.tid < oldestTid, then the object corresponding to that node is an expired object). Once an expired object $I_{e}$ is found, perform as the following steps:

Eliminate the object $I_{e}$ from the cache and its relevant information from fdHash.

$$
\text { Used }=\text { Used }-\operatorname{Size}\left(I_{e}\right)
$$

Continue to search for the next expired object; and repeat the above steps until Used $\leq$ TotalSize or no expired objects exist in fdHash.

If Used $\leq$ TotalSize, it means that there is enough cache space to store the object $I_{n}$ after removing the expired object $I_{e}$. Store the object $I_{n}$ in the cache. The processing of the object ends in the algorithm.

If Used > TotalSize, it means there is still not enough cache space to store the object $I_{n}$ after removing all expired objects. In this case, traverse fdHash, select the least $k(k \geq 1)$ eigenvalues (when the eigenvalues are the same, select the objects containing the least tid preferentially) and find their corresponding objects $I_{1}$, $I_{2}, \ldots, I_{k}$ which meet the following three conditions:

$$
\begin{aligned}
& V\left(I_{1}, T_{j}\right) \leq V\left(I_{2}, T_{j}\right) \leq \cdots \leq V\left(I_{k}, T_{j}\right) \\
& \text { Used }-\sum_{x=1}^{k} \operatorname{Size}\left(I_{x}\right) \leq \text { TotalSize } \\
& \text { Used }-\sum_{x=1}^{k-1} \operatorname{Size}\left(I_{x}\right)>\text { TotalSize }
\end{aligned}
$$

The aging factor $L$ is assigned to the maximum value $V\left(I_{k}, T_{j}\right)$, that is,

$$
L=\max _{1 \leq x \leq k} V\left(I_{x}, T_{j}\right)=V\left(I_{k}, T_{j}\right)
$$

Remove objects $I_{1}, I_{2}, \ldots, I_{k}$ from the cache. And update the value of Used:

$$
\text { Used }=\text { Used }-\sum_{x=1}^{k} \operatorname{Size}\left(I_{x}\right)
$$

Clear away the relevant information of the objects $I_{1}$, $I_{2}, \ldots, I_{k}$ from fdHash. Add the object $I_{n}$ to the cache. The process of the object is over. The above steps are looped until all objects in transaction $T_{j}$ are processed. Return to the set of objects for the transaction request, and the processing ends. The pseudocode of the algorithm GDSFDST is shown in Tab. 2.

In the algorithm GDSF-DST, the concept of sliding window model in data stream mining is employed. The idea of the sliding window model is originated from that it is impractical to store all the transactions in the data stream with limited memory size. Besides, the large time overhead for processing all historical transactions is unacceptable. Most importantly, users are more concerned about the most recent transactions. And the application of the sliding window model can exactly address the problem of data expiration.

Table 2 GDSF-DST algorithm

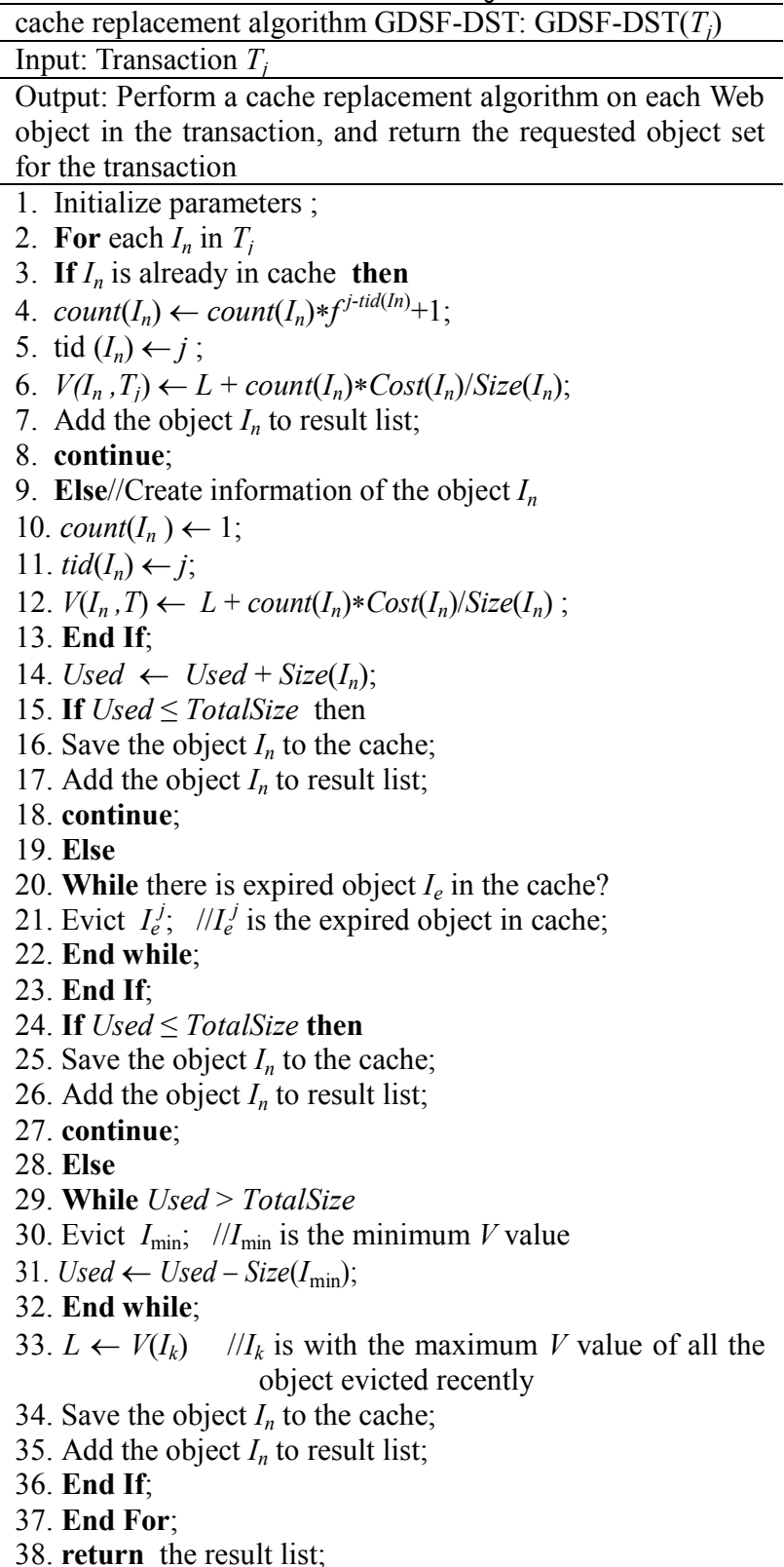

\section{EXPERIMENTS}

5.1 Experimental Design

5.1.1 Experimental Environment

In this experiment, the algorithm is implemented in Java language on the DELL PC with Microsoft Windows 7(CPU model is Intel $\mathrm{i5-7500;}$ the CPU frequency 3.4 $\mathrm{GHz}$; the capacity of the physical memory $4 \mathrm{~GB}$; the hard drive Seagate $500 \mathrm{G}$ SATA3 and the hard drive speed $7200 \mathrm{rpm})$.

\subsubsection{Experimental Data}

In this paper, the Trace Driven Simulation $[5,34]$ is adopted to simulate the algorithm. The experimental data 
are derived from the query log information recorded by the server of the spacecraft integrated test data query system. Not all the original query log information can be published, some of which is indeed unnecessary. As a result, several processed Web query log files are employed in this paper. The file naming specification is WebLog_+ log number, twenty-one query records are stored in each log file. Spacecraft integrated test data are made up of quite a few data streams, data types with huge volume of data. For ease of operation in this experiment, four representative data streams are selected. In the above $\log$ files, each record represents a query and is treated as a transaction. And a transaction usually accesses multiple Web objects. To facilitate operation in the cache replacement algorithm, each of the mentioned log files is parsed into a transaction file respectively. The transaction file naming format is TransactionFlow + the number of the corresponding $\log$ file. Each $\log$ is parsed into a transaction record. In the experiment, log data during 15 days, from September $1^{\text {th }}, 2017$ to September $15^{\text {th }}, 2017$ are employed. Since the log data statistics tables will occupy too much space, they are omitted here.

\subsubsection{Performance Indicators}

The commonly used indicators to measure the performance of the cache replacement algorithm [22] mainly include Hit Ratio (HR), Byte Hit Ratio $(B H R)$ and Delay Saving Radio (DSR). In this paper, Hit Radio $(H R)$ and Byte Hit Ratio $(B H R)$, the most commonly used ones, are adopted.Hit rate is an indication of user perceived lag, while byte hit rate is an indication of the amount of network traffic [35]. The total number of the requested Web objects is represented by $N . S_{i}$ represents the size (the number of bytes) of the ith requested Web object. $\sigma_{i}$ indicates whether the $i^{\text {th }}$ object is hit in the cache. If it is hit, $\sigma_{i}=1$; otherwise $\sigma_{i}=0 . \mathrm{L}_{\mathrm{i}}$ represents the download time required to obtain the object $\mathrm{i}$ from the original server. Definitions are as follows:

Hit Ratio $(H R)$ : The percentage for the number of the objects hit in the cache and the total number of the objects requested.

The calculation formula is: $H R=\frac{\sum_{i=1}^{N} \sigma_{i}}{N}$

Byte Hit Ratio (BHR): The percentage for the number of bytes of the objects hit in the cache and the total number of bytes of the objects requested.

The calculation formula is: $B H R=\frac{\sum_{i=1}^{N} \sigma_{i} S_{i}}{\sum_{i=1}^{N} S_{i}}$

\subsection{Experimental Analysis}

From the previous algorithm description, it can be seen that the parameters used in both GDSF and GDSFDST -- the aging factor $L$, the frequency count $f_{\text {freq }}\left(I_{n}\right)$ of the object, the decay support amount $f_{\mathrm{d}}\left(I_{n}, T_{j}\right)$ of the object and the size $\operatorname{Size}\left(I_{n}\right)$ of the object -- are defined clearly. However, measuring the cost $\operatorname{Cost}\left(I_{n}\right)$ of retrieving the object is complicated. The cost refers to anything required to obtain the object from the original server, such as the time delay, the expenditure (paid objects or paid periodic lines), the number of hops, etc., or a combination of multiple ones. In practice, the value of the $\operatorname{Cost}(I)$ is often defined according to the target of the caching system. According to the reference [26], to maximize $H R$, let $\operatorname{Cost}(I)=1$. And to maximize $B H R$, let $\operatorname{Cost}(I)=2+$ $\operatorname{Size}(I) / 536$. In the spacecraft integrated test data query system, the original data are stored on the same server, and the data server and the Web server are in the intranet environment. Therefore, the time cost of retrieving different objects from the original server is not very different, and there is no need to consider the economic cost. In this case, the value of $\operatorname{Cost}(I)$ is defined as 1 so as to achieve a better hit ratio.

\subsubsection{The Influence of Decay Factor $f$ on Hit Ratio and Byte Hit Ratio}

Before considering the influence of decay factor $f$ on the performance of the algorithm, the influence of the sliding time window size STW SIZE should be shielded first. The actual query feature of Spacecraft integrated test data query system is that users usually query data between 8 am and 8:30 pm. When STW_SIZE is set to 13 hours, there are no expired transactions within a day, that is, the sliding time window does not work. Therefore, in the experiment in this section, STW_SIZE is set to 13 hours. The literature [22] demonstrates that a better hit ratio can be achieved in the cache replacement algorithm with the relative capacity of the cache reaching $20 \%$. Therefore, in this section, the relative size of the cache capacity TotalSize is set to $20 \%$ of the total amount of access. The algorithm GDSF-DST is experimented with daily log data from September $1^{\text {th }}, 2017$ to September 10 ${ }^{\text {th }}, 2017$. When the decay factor $f$ is respectively set to $0.94,0.95,0.96$, $0.97,0.98,0.99,0.991,0.992,0.993,0.994,0.995,0.996$, 0.997, 0.998, 0.999 and 1, the values of $H R$ and $B H R$ are shown in Fig. 1 and Fig. 2 respectively. In Fig. 1 and Fig. 2 , the abscissa represents the values of the decay factor $f$ while the ordinate represents the values of the hit ratio and those of the byte hit ratio respectively. Obviously, in Fig. 1, the best hit ratio can be obtained with values of the decay factor $f$ in the range [0.996, 0.997]. When the values of $f$ are in the range [0.94, 0.996], the hit ratio increases with the increase of the values of $f$. When the values of $f$ are in the range $[0.997,1]$, the hit ratio decreases with the increase of the values of $f$. The relationship between the average hit ratio and the values of $f$ also presents the same rule. In Fig. 2, when $f=0.996$ or $f=0.997$, the byte hit ratio is the maximum. When the values of $f$ are in the range [0.94, 0.996], the byte hit ratio increases with the increase of the values of $f$. When the values of $f$ are in the range $[0.997,1]$, the byte hit ratio decreases with the increase of the values of $f$. The relationship between the average byte hit ratio and the values of $f$ also presents the same rule. When the value of $f$ changes, hit ratio and byte hit ratio have the same trend. The main reason why the decay factor $f$ has a significant impact on both the hit ratio and the byte hit ratio of the algorithm is that the data queried by the users of the spacecraft integrated test query system are of strong 
temporal locality. And the decay factor $\mathrm{f}$ can speed up the reduction of the eigenvalues of long-history data, so it can adapt to the user query behavior characteristics of the spacecraft integrated test query system better.

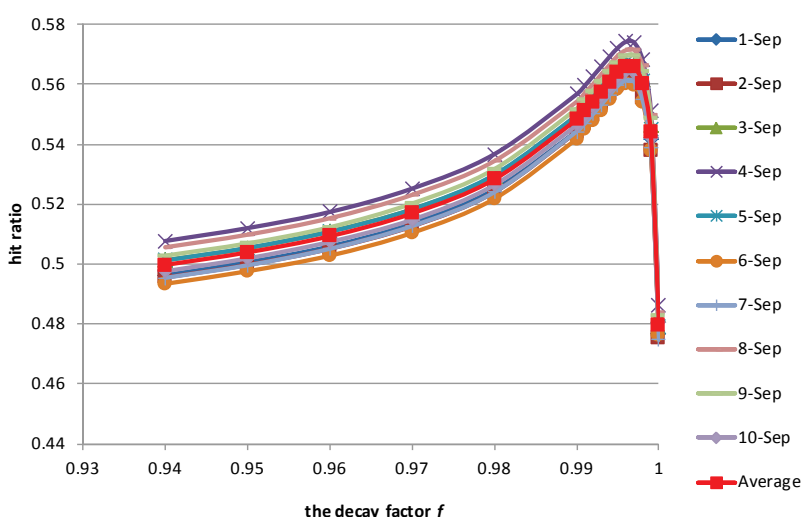

Figure 1 The relationship between $H R$ and $f$

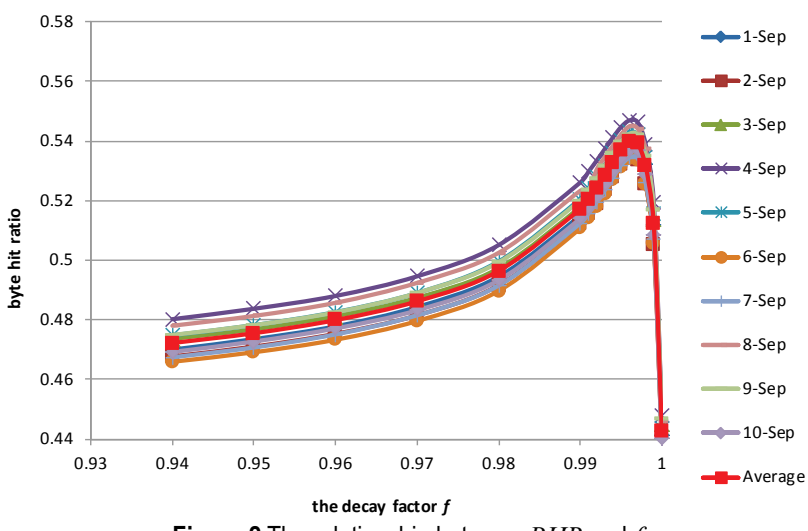

Figure 2 The relationship between $B H R$ and $f$

\subsubsection{The Influence of the Sliding Window Size $S T W_{-} S I Z E$ on Hit Ratio and Byte Hit Ratio}

Through the previous experimental analysis, it can be seen that the best hit ratio can be achieved in GDSF-DST when the values of $f$ are in the range [0.996, 0.997]. In this section, let $f=0.996$. And the relative size of the cache capacity TotalSize is still set to $20 \%$ of the total amount of access. The algorithm GDSF-DST is experimented with daily $\log$ data from September $1^{\text {th }}$, 2017 to September $10^{\text {th }}, 2017$. On the basis of the query characteristics of the system described above, when $S T W$ SIZE is greater than or equal to 13 hours, no expired transactions exist. Therefore, in this experiment, $S T W$ SIZE is set to $13 \mathrm{~h}, 12 \mathrm{~h}, 11 \mathrm{~h}, 10 \mathrm{~h}, 9 \mathrm{~h}, 8 \mathrm{~h}, 7 \mathrm{~h}, 6$ h, 5 h, 4 h, $3 \mathrm{~h}, 2 \mathrm{~h}, 1 \mathrm{~h}, 30 \mathrm{~m}, 20 \mathrm{~m}, 10 \mathrm{~m}, 9 \mathrm{~m}, 8 \mathrm{~m}, 7 \mathrm{~m}$, $6 \mathrm{~m}, 5 \mathrm{~m}, 4 \mathrm{~m}, 3 \mathrm{~m}$, and $2 \mathrm{~m}$. (h stands for hours, and $\mathrm{m}$ stands for minutes).

Tab. 3 presents the experimental data of average hit ratio and $S W$ SIZE and Tab. 4 presents the experimental data of average byte hit ratio and SW SIZE. Fig. 3, drawn from the data in Tab. 3, presents the relationship between the hit ratio and the size of STW SIZE, in which the ordinate indicates the hit ratio and the abscissa indicates the sliding window size STW SIZE. The units of $S T W$ SIZE are minutes. In order to show the changes more clearly, this plots the logarithm to base 10 of the values on the abscissa. Fig. 4, drawn from the data in Tab. 4 , presents the relationship between the byte hit ratio and the size of $S T W$ SIZE, in which the ordinate indicates the byte hit ratio and the abscissa indicates the sliding window size STW SIZE. The units of STW SIZE are minutes. Evidently, in Fig. 3 and Fig. 4, with the sliding window size declining from 10 hours to 8 minutes, GDSF-DST can achieve great hit ratio and byte hit ratio, and the performance index remains stable. When the size of the sliding window is greater than 10 hours, as the size of the sliding window increases, both hit ratio and byte hit ratio decrease, which indicates that the performance of the algorithm can be improved to a certain extent by adding a sliding window to determine expired transactions.

When the sliding window is less than 2 minutes, both the hit ratio and the byte hit ratio of the algorithm become extremely unstable and rapidly decrease. This is because the time is too short, and many other transactions related to the ones being queried by users are also thrown out prematurely; while when the sliding window size reaches 8 minutes, the algorithm GDSF-DST can achieve better hit ratio and byte hit ratio and the performance index remains stable. It shows that the time of the transactions related to the transaction queried by users generally maintains between 2 minutes and 8 minutes. And often in some small period of time there are some objects accessed at high frequency.

Table 3 The relationship between $H R$ and STW SIZE

\begin{tabular}{|c|c|c|c|c|c|c|c|c|c|c|c|c|}
\hline STW SIZW / min & 780 & 720 & 660 & 600 & 540 & 480 & 420 & 360 & 300 & 240 & 180 & 120 \\
\hline The average & 0.565 & 0.566 & 0.567 & 0.567 & 0.567 & 0.567 & 0.567 & 0.567 & 0.567 & 0.567 & 0.567 & 0.567 \\
\hline hit rat & 983 & 099 & 023 & 023 & 023 & 023 & 023 & 023 & 023 & 023 & 023 & 023 \\
\hline$S W \_S I Z W / \min$ & 60 & 30 & 20 & 10 & 9 & 8 & 7 & 6 & 5 & 4 & 3 & 2 \\
\hline The average & 0.567 & 0.567 & 0.567 & 0.567 & 0.567 & 0.567 & 0.566 & 0.566 & 0.566 & 0.566 & 0.566 & 0.562 \\
\hline hit ratio & 023 & 023 & 023 & 023 & 023 & 023 & 999 & 958 & 958 & 955 & 732 & 552 \\
\hline
\end{tabular}

Table4 The relationship between $B H R$ and STW SIZE

\begin{tabular}{|c|c|c|c|c|c|c|c|c|c|c|c|c|}
\hline$S T W$ SIZW / min & 780 & 720 & 660 & 600 & 540 & 480 & 420 & 360 & 300 & 240 & 180 & 120 \\
\hline The av & 0.539 & 0.539 & 0.540 & 0.540 & 0.540 & 0.540 & 0.540 & 0.540 & 0.540 & 0.540 & 0.540 & 0.540 \\
\hline byte hit ratio & 581 & 693 & 668 & 668 & 668 & 668 & 668 & 668 & 668 & 668 & 668 & 668 \\
\hline$S W \_S I Z W / \min$ & 60 & 30 & 20 & 10 & 9 & 8 & 7 & 6 & 5 & 4 & 3 & 2 \\
\hline The average & 0.540 & 0.540 & 0.540 & 0.540 & 0.540 & 0.540 & 0.540 & 0.540 & 0.540 & 0.540 & 0.540 & 0.538 \\
\hline byte hit ratio & 668 & 668 & 668 & 668 & 668 & 668 & 653 & 606 & 606 & 614 & 667 & 913 \\
\hline
\end{tabular}

As the size of the sliding window is reduced from 10 hours to 8 minutes, the hit ratio and the byte hit ratio remain stable. Because a query usually involves a wide range of objects, transactions in a short time can contain most objects, which makes a large number of the objects non-expired. The smaller sliding windows are, the fewer 
the stored transactions are, thereby reducing the spatial complexity of the algorithm. Therefore, under the premise of ensuring the performance of the algorithm, the smaller time window should be selected. In Fig. 3 and Fig. 4, the red data point is the one of the sliding window size as 10 minutes, and the performance of the algorithm is stable before and after the point. Therefore, for the workload of the spacecraft integrated test data query system, the sliding window size can be selected as 10 minutes.

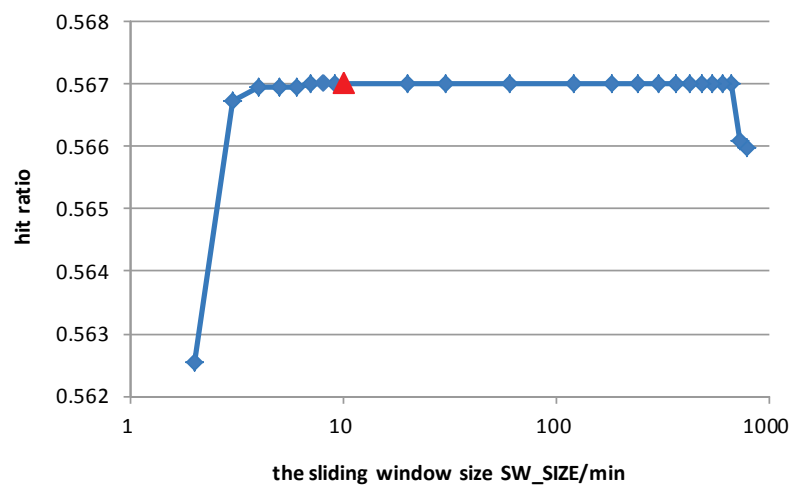

Figure 3 The relationship between hit ratio and STW_SIZE size

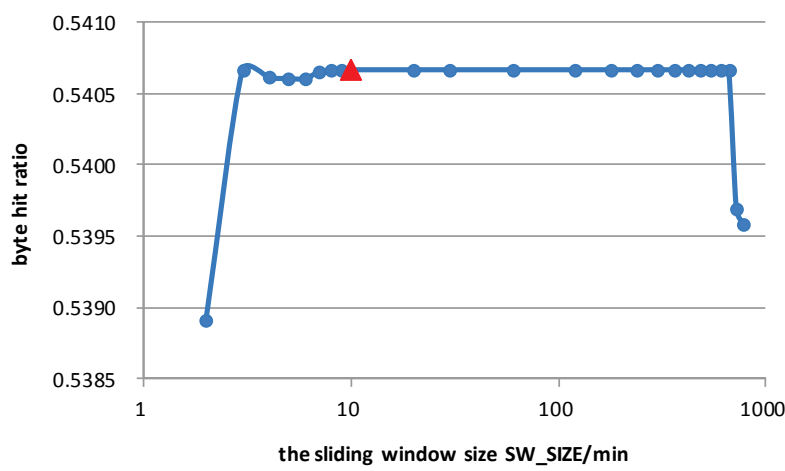

Figure 4 The relationship between byte hit ratio and STW_SIZE size

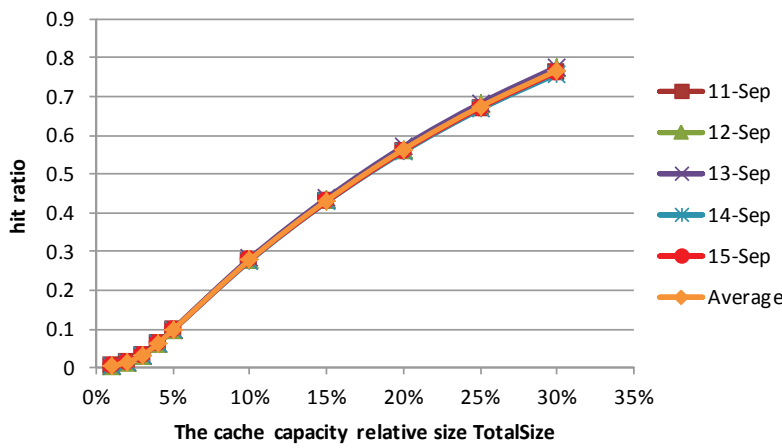

Figure 5 The relationship between hit ratio and cache capacity

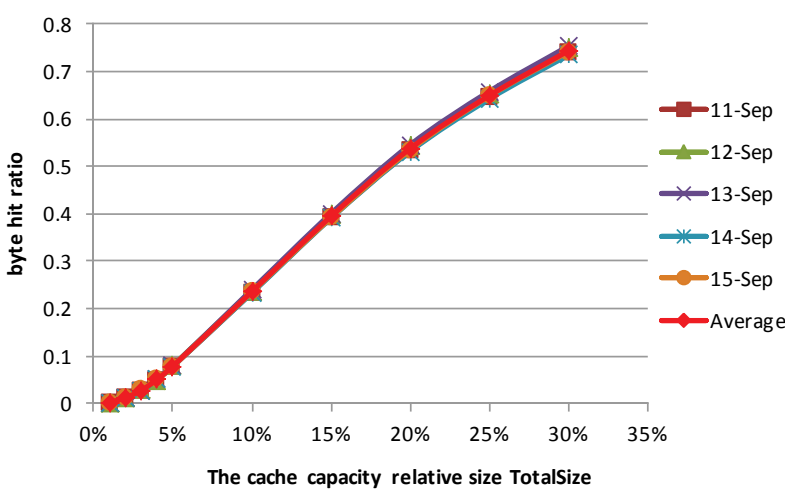

Figure 6 The relationship between byte hit ratio and cache capacity

\subsubsection{The Influence of the Cache Capacity TotalSize on Hit Ratio and Byte Hit Ratio}

Based on the previous analysis, in this section, the sliding window size $S T W$ SIZE is set to 10 minutes, and the decay factor $f$ is set to 0.996 . The cache capacity relative size TotalSize is set to $1 \%, 2 \%, 3 \%, 4 \%, 5 \%, 10 \%$, $15 \%, 20 \%, 25 \%$, and $30 \%$, respectively, and then experiments are carried out. The log data used for the experiments are the $\log$ records during 5 days, from September $11^{\text {th }}, 2017$ to September $15^{\text {th }}, 2017$. In Fig. 5, the ordinate represents the value of the hit ratio and the abscissa indicates the relative capacity of the cache. In Fig 6 , the ordinate represents the value of the byte hit ratio and the abscissa indicates the relative capacity of the cache. It can be seen from the figures that the hit ratio and the byte hit ratio increase rapidly with the increase of the cache capacity TotalSize. When the relative capacity of the cache reaches $30 \%$, the hit ratio and the byte hit ratio can exceed $70 \%$.

\subsubsection{Comparison with the Classical Algorithms}

In this section, the sliding window size $S T W$ SIZE in the algorithm GDSF-DST is set to 10 minutes and the decay factor $f$ is set to 0.996 . The $\log$ data used for the experiments are the $\log$ records during 5 days, from September $11^{\text {th }}, 2017$ to September $15^{\text {th }}, 2017$. The relative cache capacity TotalSize is set to $1 \%, 2 \%, 3 \%, 4 \%$, $5 \%, 10 \%, 15 \%, 20 \%, 25 \%$, and $30 \%$, respectively, and experiments are carried out respectively on algorithms GDSF-DST, GDSF, LFU, LFU-DA and LRU.

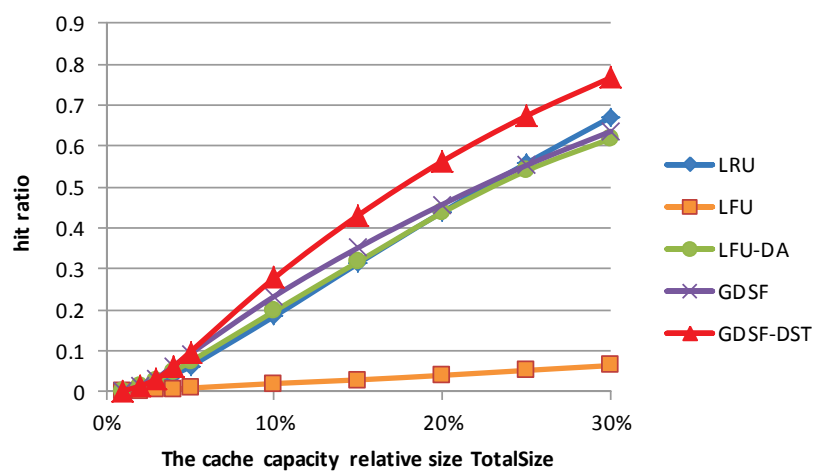

Figure 7 Comparison of hit ratios for multiple algorithms

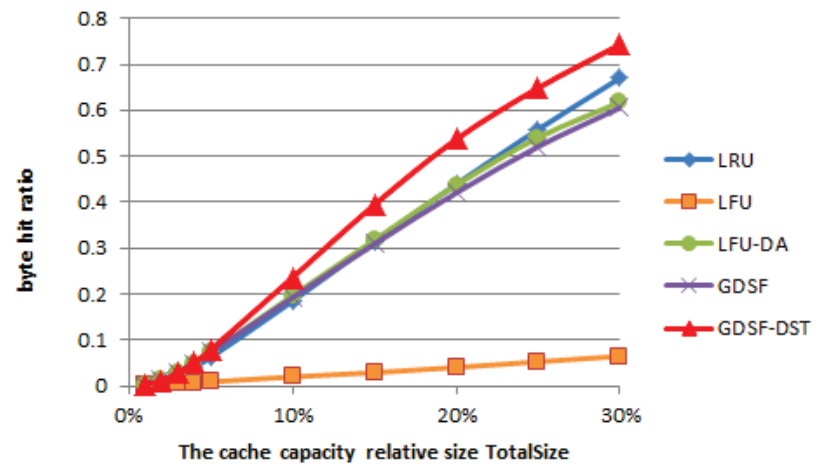

Figure 8 Comparison of byte hit ratios for multiple algorithms

Fig. 7 shows the curves of hit ratio of each algorithm varying with the cache capacity. Fig. 8 shows the curves of the byte hit ratio of each algorithm varying with the 
cache capacity. Because the decay factor $f$ and the sliding window mechanism are introduced for the users' query behavior characteristics of the spacecraft integrated test query system, both the hit ratio and the byte hit ratio in GDSF-DST algorithm are better than those in other algorithms (Fig. 7 and Fig. 8).

\section{CONCLUSIONS}

(1) In this paper, the characteristics of data in spacecraft integration test data query system and users' query behaviors are analyzed. Under the premise of full investigation of the cache technology research progress, combined with the relevant technology of Web data stream mining, a cache replacement algorithm, GDSFDST, is designed for Spacecraft integrated testing data query system.

(2) The specific process of the algorithm implementation is presented and the Trace Driven Simulation experiment is performed with the query log data in the actual runtime environment.

(3) The newly-designed algorithm is fully analyzed and compared with the existing typical cache replacement algorithms, and it is proved that the algorithm has better performance.

The deficiency in this paper and further work is to evaluate the validity of the algorithm with Data Envelopment Analysis (DEA). The method proposed in this paper is only for the spacecraft integration test data query. In future, we will collect data in other fields except spacecraft comprehensive test, such as stock trading, video websites, news websites, and some other data applications with large volume of data, rapid changes and the latest data changes which public users are more concerned about. And then we will discuss the application and expansion of GDSF-DST algorithm proposed in this paper in these fields. The new algorithm proposed in this paper is integrated with the algorithms of machine learning to further improve the performance index of cache replacement strategy and to verify the applicability of the new intelligent algorithms in other environments.

\section{ACKNOWLEDGEMENTS}

This work was supported by the National Natural Science Foundation of China under Grant Nos. U1736116 and 61300007, the Fundamental Research Funds for the Central Universities of China under Grant Nos. YWF-15GJSYS-106 and YWF-14-JSJXY-007, and the Project of the State Key Laboratory of Software Development Environment of China under Grant Nos. SKLSDE 2015ZX-09 and SKLSDE-2014ZX-06.

\section{REFERENCES}

[1] Djurovic, D., Bulatovic, M., Sokovic, M., \& Stoic, A. (2015). Measurement of maintenance excellence/Mjerenje izvrsnosti odrzavanja. Tehnicki Vjesnik-Technical Gazette, 22(5), 1263-1269. https://doi.org/10.17559/TV-20140922094945

[2] Blagojević, M., Rakić, D., Topalović, M., \& Živković, M. (2016). Optical coordinate measurements of parts and assemblies in automotive industry. Tehnicki vjesnik/Technical Gazette, 23(5), 1541-1546. https://doi.org/10.17559/TV-20130918160442
[3] Lv, J., Ma, S., Li, X., et al. (2015). A high order collaboration and real time formal model for automatic testing of safety critical systems. Frontiers of Computer Science, 9(4), 495-510. https://doi.org/10.1007/s11704-015-2254-y

[4] Gao, S. W., Lv, J. H., Du, B. L., et al. (2015). Balancing Frequencies and Fault Detection in the In-Parameter-Order Algorithm.Journal of Computer Science and Technology, 30(5), 957-968. https://doi.org/10.1007/s11390-015-1574-6

[5] Kastaniotis, G., Maragos, E., Douligeris, C., et al. (2012).Using data envelopment analysis to evaluate the efficiency of Web caching object replacement strategies. Journal of Network and Computer Applications, 35(2), 803817. https://doi.org/10.1016/j.jnca.2011.11.013

[6] Podlipnig, S. \& Böszörmenyi, L. (2003). A survey of web cache replacement strategies. ACM Computing Surveys (CSUR), 35(4), 374-398. https://doi.org/10.1145/954339.954341

[7] Aggarwal, C., Wolf, J. L., \& Yu, P. S. (1999). Caching on the world wide web. IEEE Transactions on Knowledge and data Engineering, 11(1), 94-107. https://doi.org/10.1109/69.755618

[8] Geetha, K. \& Gounden, N. A. (2017). Dynamic Semantic LFU Policy with Victim tracer (DSLV): A Customizing Technique for Client Cache. Arabian Journal for Science and Engineering, 42(2), 725-737. https://doi.org/10.1007/s13369-016-2287-z

[9] Wang, S., Li, Z., Chao, W., et al. (2012). Applying adaptive over-sampling technique based on data density and costsensitive SVM to imbalanced learning. International Joint Conference on Neural Networks. IEEE, 2, 1-8. https://doi.org/10.1109/IJCNN.2012.6252696

[10] Wang, S., Li, Z., Zhang, X. (2013). Bootstrap Sampling Based Data Cleaning and Maximum Entropy SVMs for Large Datasets. IEEE, International Conference on TOOLS with Artificial Intelligence. IEEE, 1(11), 1151-1156. https://doi.org/10.1109/ICTAI.2012.164.

[11] Li, Y., Zhou, G., Nie, B. (2017). Improving Web Performance in Home Broadband Access Networks. Wireless Personal Communications, 92(3), 925-940. https://doi.org/10.1007/s11277-016-3585-1

[12] Ali, W., Shamsuddin, S. M., \& Ismail, A. S. (2012). Intelligent Web proxy caching approaches based on machine learning techniques. Decision Support Systems. 53(3), 565-579. https://doi.org/10.1016/j.dss.2012.04.011

[13] Ali, W., Shamsuddin, S. M., \& Ismail, A. S. (2012).Intelligent Naïve Bayes-based approaches for Web proxy caching. Knowledge-Based Systems, 31, 162-175. https://doi.org/10.1016/j.knosys.2012.02.015

[14] Songwattana, A., Theeramunkong, T., \& Vinh, P. C. (2014). A learning-based approach for web cache management. Mobile Networks and Applications, 19(2), 258-271. https://doi.org/10.1007/s11036-014-0498-7

[15] Lee, C. (2013). Streaming media service based on fuzzy similarity in wireless mobile networks. The Journal of Supercomputing, 65(1), 86-105. https://doi.org/10.1007/s11227-012-0778-6

[16] Sajeev, G. P. \& Sebastian, M. P. (2013). Building semiintelligent web cache systems with lightweight machine learning techniques. Computers \& Electrical Engineering, 39(4), 1174-1191. https://doi.org/10.1016/j.compeleceng.2013.02.005

[17] Romano, S. \& ElAarag, H. (2011). A neural network proxy cache replacement strategy and its implementation in the Squid proxy server. Neural computing and Applications, 20(1), 59-78. https://doi.org/10.1007/s00521-010-0442-0

[18] Rashkovits, R. (2016). Preference-based content replacement using recency-latency tradeoff. World Wide Web, 19(3), 323-350. https://doi.org/10.1007/s11280-014-0313-1 
[19] Pan, S., Chong, Y., Xu, Z., et al. (2017). An enhanced active caching strategy for data-intensive computations in distributed GIS. The Journal of Supercomputing, 73(10), 4324-4346. https://doi.org/10.1007/s11227-017-2012-z

[20] Sun, X. C., Wang, Z. J., Chu, H., et al. (2016). An Efficient Resource Management Algorithm for Information Centric Networks. Journal of Internet Technology, 17(5), 10071015. https://doi.org/10.6138/JIT.2016.17.5.20160111

[21] Chen, J., Lee, V. C. S., Liu, K., et al. (2017). Efficient Cache Management for Network-Coding-Assisted Data Broadcast. IEEE Transactions on Vehicular Technology. 66(4), 3361-3375. https://doi.org/10.1109/TVT.2016.2589460

[22] Kastaniotis, G., Maragos, E., Dimitsas, V., et al. 2007. Web Proxy caching object replacement: Frontier analysis to discover the good-enough algorithms. Proceedings of the 2007 15th International Symposium on Modeling, Analysis, and Simulation of Computer and Telecommunication Systems. IEEE Computer Society, 132-137. https://doi.org/10.1109/MASCOTS.2007.68

[23] Gao, S. W., Lv, J. H., Wuniri, Q. Q. G., et al. (2015). Spacecraft Test Requirement Description and Automatic Generation Method. Journal of Beijing University of Aeronautics and Astronautics, 41(7), 1275-1286. https://doi.org/10.13700/j.bh.1001-5965.2014.0762

[24] Chen, H. (2007). Design and Implementation of Integration Test Data Platform for Manned Spacecraft. PhD. Thesis, Beijing University of Aeronautics and Astronautics, Beijing.

[25] Young, N. (1994). Thek-server dual and loose competitiveness for paging. Algorithmica, 11(6), 525-541. https://doi.org/10.1007/BF01189992

[26] Cao, P. \& Irani, S. (1997). Cost-aware www proxy caching algorithms. Usenix symposium on internet technologies and systems, 12(97), 193-206.

[27] Cherkasova, L. (1998). Improving www proxies performance with greedy-dual-size-frequency caching policy. Hp Technical Report.

[28] Ferrara, E., De Meo, P., Fiumara, G., et al. (2014). Web data extraction, applications and techniques: A survey. Knowledge-based systems, 70, 301-323. https://doi.org/10.1016/..knosys.2014.07.007

[29] Li, H. F. \& Lee ,S. Y. (2009). Mining frequent itemsets over data streams using efficient window sliding techniques. Expert Systems with Applications, 36(2), 14661477. https://doi.org/10.1016/j.eswa.2007.11.061

[30] Thanh Lam, H. \& Calders, T. (2010). Mining top-k frequent items in a data stream with flexible sliding windows. Proceedings of the $16^{\text {th }}$ ACM SIGKDD international conference on Knowledge discovery and data mining. ACM, 283-292. https://doi.org/10.1145/1835804.1835842

[31] Mahanti, A., Eager, D., \& Williamson, C. (2000). Temporal locality and its impact on Web proxy cache performance. Performance Evaluation, 42(2),187-203. https://doi.org/10.1016/S0166-5316(00)00032-8

[32] Giannella, C., Han, J., Pei, J., et al. (2003). Mining frequent patterns in data streams at multiple time granularities. Next generation data mining, 212, 191-212.

[33] Chang, J. H. \& Lee, W. S. (2003). Finding recent frequent itemsets adaptively over online data streams. Proceedings of the ninth $A C M$ SIGKDD international conference on Knowledge discovery and data mining, ACM, 487-492. https://doi.org/10.1145/956750.956807

[34] Patil, J. B. \& Pawar, B. V. (2007). Trace driven simulation of GDSF\# and existing caching algorithms for web proxy servers. Proceedings of the $6^{\text {th }}$ WSEAS International Conference on Data Networks, Communications and Computers (DNCOCO 2007), Trinidad and Tobago. 378-
384.

[35] Romano, S. \& ElAarag, H. (2012). A quantitative study of Web cache replacement strategies using simulation. Simulation, 88(5), 507-541.

https://doi.org/10.1007/978-1-4471-4893-7_4

\section{Contact information:}

Jianhai DU, PhD Student

School of Computer Science and Technology,

Beijing University of Aeronautics and Astronautics

Beijing, 100191, China

E-mail: dujianhai6@163.com

Shiwei GAO, Engineer

Beijing Institute of Aerospace Control Devices

Beijing, 100039, China

E-mail: ge89@163.com

Jianghua LV, Associate Professor

(Corresponding author)

School of Computer Science and Technology,

Beijing University of Aeronautics and Astronautics

Beijing, 100191, China

E-mail: jianghualvpaper@126.com

Qianqian LI,

School of Computer Science and Technology,

Beijing University of Aeronautics and Astronautics

Beijing, 100191, China

E-mail: paper135789@163.com

Shilong MA, Professor

School of Computer Science and Technology,

Beijing University of Aeronautics and Astronautics

Beijing, 100191, China

E-mail: shilongma8@163.com 